\title{
THE PLANETARY THEORIES AND THE PRECESSION OF THE ECLIPTIC
}

\author{
P. BRETAGNON \\ Bureau des longitudes, URA707 \\ 77 , avenue Denfert-Rochereau \\ F-75014 Paris, France
}

\section{Introduction}

In this paper, I give the present state of the analytical planetary theories by describing the general theories and the secular variation theories, the variations of the ecliptic with respect to the ecliptic $\mathrm{J} 2000$, the utilization of the analytical planetary theories in the calculation of the precessionnutation of the equator and in the calculation of the expressions of transformation between the barycentric and geocentric reference systems. At last, I describe the construction of new planetary theories undertaken at the Bureau des longitudes.

The analytical planetary theories arise in two forms: the general theories give, with a low accuracy, the variations of the elements of the planets over several million years; the secular variation theories reach a high accuracy over time spans of a few thousands of years.

In all these solutions, the motion of the planets is represented with 6 elements: $a$, the semi major axis, $\lambda$, the mean longitude and the variables $k=e \cos \varpi, h=e \sin \varpi, q=\sin \frac{i}{2} \cos \Omega$, $p=\sin \frac{i}{2} \sin \Omega$ where $e$ represents the eccentricity of the orbit, $\varpi$ the longitude of the perihelion, $i$ the inclination of the orbit about the ecliptic J2000 and $\Omega$ the longitude of the node. It is not possible to represent the variations of the planetary orbits with the elements $e, \varpi, i$ and $\Omega$ because, for most of the planets, $e$ and $i$ can reduce to zero and therefore $\varpi$ and $\Omega$ are not continuous functions.

\section{General planetary theories}

In the general theories, the planetary elements are represented as Fourier series with an additive constant for the semi major axis and a linear function in time for the mean longitude.

$$
\begin{aligned}
a_{i}= & a_{i 0}+\sum_{\Phi^{*}} A_{i, \Phi^{*}} \cos \Phi^{*}+\sum_{\Phi} A_{i, \Phi} \cos \Phi \\
\lambda_{i}= & \lambda_{i 0}+\lambda_{i 1} t+\sum_{\Phi^{*}} B_{i, \Phi^{*}} \sin \Phi^{*}+\sum_{\Phi} B_{i, \Phi} \sin \Phi \\
k_{i}= & \sum_{k=1}^{8} \lambda_{i k} M_{k} \cos \psi_{k}+\sum_{k=1}^{8} M_{i, \psi_{k}} \cos \psi_{k}+\sum_{\Phi^{*}} M_{i, \Phi^{*}} \cos \Phi^{*}+\sum_{\Phi} M_{i, \Phi} \cos \Phi \\
h_{i}= & \sum_{k=1}^{8} \lambda_{i k} M_{k} \sin \psi_{k}+\sum_{k=1}^{8} M_{i, \psi_{k}} \sin \psi_{k}+\sum_{\Phi^{*}} M_{i, \Phi^{*}} \sin \Phi^{*}+\sum_{\Phi} M_{i, \Phi} \sin \Phi \\
q_{i}= & \sum_{k=1}^{8} \mu_{i k} N_{k} \cos \theta_{k}+\sum_{k=1}^{8} N_{i, \theta_{k}} \cos \theta_{k}+\sum_{\Phi^{*}} N_{i, \Phi^{*}} \cos \Phi^{*}+\sum_{\Phi} N_{i, \Phi} \cos \Phi \\
p_{i}= & \sum_{k=1}^{8} \mu_{i k} N_{k} \sin \theta_{k}+\sum_{k=1}^{8} N_{i, \theta_{k}} \sin \theta_{k}+\sum_{\Phi^{*}} N_{i, \Phi^{*}} \sin \Phi^{*}+\sum_{\Phi} N_{i, \Phi} \sin \Phi
\end{aligned}
$$

The $\Phi$ arguments are linear combinations of the angles $\bar{\lambda}_{j}$ (coefficient $r_{j}$ ), $\psi_{j}$, and $\theta_{j} . \bar{\lambda}_{j}$ represents the argument of the planetary longitude $\lambda_{0}+\lambda_{1} t, \psi_{j}$ the argument of the Lagrange solution in eccentricity, $\theta_{j}$ the argument of the Lagrange solution in inclination connected to the planet $j$. The periods of the angles $\lambda_{j}$ are the periods of the planets included between 88 days and 165 
years if we consider 8 planets from Mercury to Neptune. The periods of the angles $\psi$ and $\theta$ are included between 46000 years and 2 million years. The matrices $\left(\lambda_{i k}\right)$ and $\left(\mu_{i k}\right)$ are the matrices of the eigenvectors of the Lagrange solution and the part of the solutions which contains $\left(\lambda_{i k}\right)$ and $\left(\mu_{i k}\right)$ is the Lagrange-Laplace solution of degree 1 in eccentricity or inclination; the other terms are of higher degree. In these expressions, the $\Phi^{*}$ arguments correspond to the case in which all the coefficients of the $\bar{\lambda}_{j}$ are zeros, that is to say to the long period terms. We have hereunder, for example, the long period parts of the variable $k$ of the Earth with the periods in years; $h$ is represented by a similar expression with sines instead of cosines. The time $t$ is counted in thousands of Julian years from $\mathrm{J} 2000$.

$\begin{array}{rlr}k= & 0.004260 \cos (1.561714+0.0272157 t) & 230866 \\ & +0.016135 \cos (3.471568+0.0361472 t) & 173822 \\ & +0.010246 \cos (5.610357+0.0838008 t) & 74978 \\ & -0.013399 \cos (5.414083+0.0867938 t) & 72392 \\ & +0.018173 \cos (0.540233+0.0208860 t) & 300832 \\ & +0.001439 \cos (2.286358+0.1364670 t) & 46042 \\ & +0.000598 \cos (2.066687+0.0152880 t) & 410988 \\ & +0.000013 \cos (1.302756+0.0032650 t) & 1924406 \\ & +0.000182 \cos (2.704153+0.0849704 t) & 73946 \\ & +0.000276 \cos (5.926229+0.0916930 t) & 68524 \\ & -0.003367 \cos (4.901937+0.0818946 t) & 76723 \\ & +0.000378 \cos (5.217809+0.0897868 t) & 69979 \\ & -0.002354 \cos (6.122503+0.0887000 t) & 70836 \\ & +0.000857 \cos (5.098211+0.0789016 t) & 79633 \\ & +0.000599 \cos (5.806631+0.0808078 t) & 77755 \\ & -0.000174 \cos (2.310136+0.0305229 t) & 205852 \\ & +0.001007 \cos (2.723146+0.0328400 t) & 191327 \\ & -0.000124 \cos (3.725634+0.0913001 t) & 68819 \\ & -0.000337 \cos (2.583195+0.0335454 t) & 187304 \\ & +\ldots \\ & + \text { periodic terms }\end{array}$

These expressions keep an accuracy of about $10^{-4}$ for $k$ and $h$ and therefore for the eccentricity over several million years.

The long periods of this solution being in between 46000 years and 2 million years, it is possible to develop the sines and the cosines with respect to time and to represent the solution as polynomials in time over 1000 years. We get the following polynomials here limited to the fifth degree

$$
\begin{gathered}
k=-.003742-.0008287 t+.0000270 t^{2}+11588 \times 10^{-10} t^{3}-231 \times 10^{-10} t^{4}-7 \times 10^{-10} t^{5} \\
h=-.016286-.0006087 t-.0000346 t^{2}+8596 \times 10^{-10} t^{3}+312 \times 10^{-10} t^{4}-5 \times 10^{-10} t^{5}
\end{gathered}
$$

In the same way, we have the long period parts of the variable $q$ of the Earth with the period in years and a similar expression for $p$ with sines. The accuracy of the inclination of the ecliptic about the ecliptic . 12000 given by such expressions is about $10^{\prime \prime}$ over several million years.

$$
\begin{array}{rlr}
q= & 0.006037 \cos (0.247373-0.0272026 t) & 230977 \\
& +0.002582 \cos (5.369126-0.0328269 t) & 191404 \\
& +0.010016 \cos (4.366638-0.0912870 t) & 68829 \\
& +0.003802 \cos (4.878784-0.0863878 t) & 72732 \\
& +0.013775 \cos (1.877845) & \\
& +0.001405 \cos (2.210841-0.1270650 t) & 49449 \\
& -0.000866 \cos (5.554460-0.0145436 t) & 432024 \\
& -0.000650 \cos (3.548901-0.0033522 t) & 1874347 \\
& -0.000378 \cos (3.854492-0.0961862 t) & 65323
\end{array}
$$




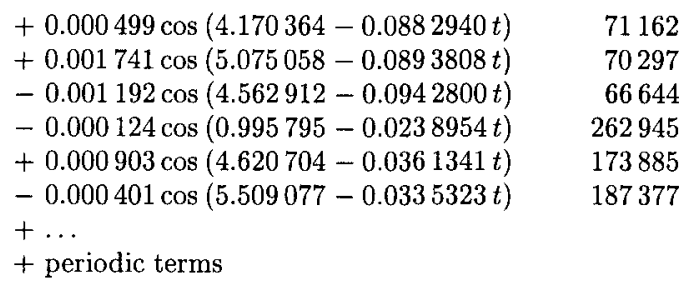

The development with respect to time of the long period terms of $q$ and $p$ gives the polynomials

$$
\begin{gathered}
q=\quad-0.0011325 t+0.00001228 t^{2}+12661 \times 10^{-10} t^{3}-147 \times 10^{-10} t^{4}-3 \times 10^{-10} t^{5} \\
p=\quad-0.0000960 t+0.00004689 t^{2}-5124 \times 10^{-10} t^{3}-245 \times 10^{-10} t^{4}+3 \times 10^{-10} t^{5}
\end{gathered}
$$

The precision of the general theories is limited because of the very large number of arguments, linear combinations of 24 components and these solutions cannot reach the level of accuracy of the present observations. On the contrary, the secular variation theories represent the motion of the planets with a very high precision.

\section{Secular variation theories}

The secular variation theories build, in a direct way, the polynomial part as well as the Fourier and Poisson series $S_{j}$, with sines and cosines, which only depend on the 8 planetary mean longitudes. In the expression

$$
x=x_{0}+x_{1} t+\ldots+x_{j+1}+S_{0}+t S_{1}+\ldots+t^{j} S_{j}
$$

with

$$
S_{j}=\sum_{i}\left(a_{j, i} \sin \left(\sum_{k=1}^{8} r_{k} \bar{\lambda}_{k}\right)+b_{j, i} \cos \left(\sum_{k=1}^{8} r_{k} \bar{\lambda}_{k}\right)\right)
$$

$x_{0}$ is an integration constant, the other quantities are calculated perturbations.

For the Earth, we obtain the polynomial parts of the variables $k, h, q$ and $p$

$$
\begin{aligned}
& k=\quad-0.0037408165-0.0008226742 t+0.0000276246 t^{2}+11696 \times 10^{-10} t^{3} \\
& -270 \times 10^{-10} t^{4}-7 \times 10^{-10} t^{5} \\
& h=\quad-0.0162844766-0.0006202965 t-0.0000338263 t^{2}+8510 \times 10^{-10} t^{3} \\
& +277 \times 10^{-10} t^{4}-5 \times 10^{-10} t^{5} \\
& q=-0.0011346887 t+0.0000123731 t^{2}+12654 \times 10^{-10} t^{3}-137 \times 10^{-10} t^{4}-3 \times 10^{-10} t^{5} \\
& p=\quad-0.0001018038 t+0.0000470200 t^{2}-5417 \times 10^{-10} t^{3}-251 \times 10^{-10} t^{4}+5 \times 10^{-10} t^{5}(3)
\end{aligned}
$$

\subsection{COMPARISON OF THE POLYNOMIAL PARTS OF THE EARTH'S ELEMENTS WITH THE GENERAL THEORIES}

It is interesting to compare the polynomials (3) with the long period terms of the general theories obtained from the developments (1) and (2) with respect to time.

For the Earth over 1000 years, the differences for the variables $k$ and $h$ and therefore for the eccentricity are about $10^{-5}$; for $q$ and $p$, the differences are about $5 \times 10^{-6}$ which corresponds to 2 arcseconds for the inclination.

In this comparison, we can see that it is easy to obtain a low accuracy secular variation solution from a general theory but, of course, the contrary is not possible. Over 1000 years, to the variables $k$ and $h$ correspond the polynomial parts of the eccentricity and of the longitude of the perihelion

$$
\begin{array}{cc}
e= & 0.0167086342-0.0004203654 t-0.0000126734 t^{2}+\ldots \\
\varpi= & 102.93734808+11612^{\prime \prime} .35290 t+53^{\prime \prime} .27577 t^{2}+\ldots
\end{array}
$$


Let us recall that, over 100000 years, the longitude of the perihelion is not a continuous function and that the secular variation solutions represent the planetary motion over only 1000 years. When the eccentricity becomes zero, we have a discontinuity of the perihelion of 180 degrees.

Therefore the secular term of 11".61235290 per year in the formula (4) is not the frequency of a periodic term with a 111605 year period reckoned from the equinox J2000 or with a 20937 year period reckoned from the equinox of date.

There are not such periods in the solar system and there is no meaning to introduce such periods in the heliocentric motion of the Earth or in the precession-nutation theory.

\subsection{ACCURACY OF THE SECULAR VARIATION THEORY OF THE EARTH}

The integration constants of the VSOP82 (Bretagnon, 1982) and VSOP87 (Bretagnon and Francou, 1988) solutions have been determined by fitting to the numerical integration DE200 (Standish, 1982) of the JPL. The comparison, between 1900 and 2000, of VSOP with DE200 shows differences of a few mas, 5 mas for the longitude of the Earth and about $10^{-8}$ for the other variables. The inclination of the true ecliptic about the ecliptic J2000 is defined with an accuracy of 3 mas but the main reason of the uncertainty of the analytical and numerical planetary solutions comes from the quality of the observations used to determine the integration constants. Thus, between DE200 and DE403, we have differences which reach 30 mas for the Earth over 1900-2000.

The accuracy of VSOP 82 is therefore consistent with the quality of the observations used in DE200.

\section{Utilization of the analytical theories in the calculation of the precession-nutation}

This accuracy of the VSOP solutions allows us to build the contributions of the Sun and the planets to the analytical precession-nutation series at the level of $0.1 \mu$ as. The perturbations of the terrestrial equator due to the Moon are calculated with an uncertainty of about $1 \mu$ as by using the analytical solution ELP. The comparison for the three Euler angles $\psi, \omega$ and $\varphi$ between the analytical precession-nutation solution SMART97 (Bretagnon et al., 1997a, 1997b) and a numerical solution using the numerical integration DE403/LE403 gives residuals are $2 \mu$ as for $\psi, 0.6 \mu$ as for $\omega$, and $2 \mu$ as for $\varphi$ over 55 years between 1968 and 2023 .

Note that, as in the analytical solution of the Sun, there is no meaning to introduce the 20937 year period term in the nutation series. One find also, in the classical nutation series, similar period arguments which are in phase every 20937 years or 10468 years such as the arguments of period 18.6 years, 6 months, one year. There is no meaning to keep 2 arguments with similar periods that it is impossible to discriminate over the 20 year time span of the high precision observations.

\section{Utilization of the analytical theories in the calculation of the expressions of trans- formation between the barycentric and geocentric reference systems}

The analytical solutions of the planetary motions are used to calculate the expressions of transformation between the barycentric and geocentric reference systems. Several solutions have been built at the first order in $c^{-2}$ where $c$ is the light velocity.

The analytical solution for TCB - TCG (Fairhead and Bretagnon, 1990) has been compared with a numerical integration of the JPL. The differences are smaller than 3.4 nanoseconds over $1900-2000$.

Fukushima has completed the analytical solution by taking into account the post-Newtonian effects and the perturbations due to the asteroids. The comparison to numerical integrations he has performed gives an accuracy of the analytical solution of 1.8 nanosecond over the time span $1980-2000$.

\section{New planetary theories}

We have seen that the VSOP82 and VSOP87 planetary solutions allow us to determine the variations of the rigid Earth equator with an accuracy of $1 \mu$ as and the relation between $T C B$ and $T C G$ with an accuracy of 1.8 nanosecond. 
Nevertheless, it is useful to have today better analytical planetary theories, specially to provide very precise relations between the barycentric and geocentric coordinates, relations which are essential to the reduction of the observations VLBI, SLR, LLR.

Moisson (Bureau des longitudes) has undertaken the construction of a new planetary theory by using recent values of the planetary masses. The first step of the work gives solutions three times more precise than VSOP82. The improvement is particularly important for the variables $q$ and $p$ which define the inclination and the longitude of the node of the ecliptic. In a comparison with DE403, the residuals in inclination do not exceed 0.1 mas over $1900-2000$ which is 30 times more precise than the VSOP82 solution. His new solution shall be expressed in two forms: as a function in Barycentric dynamical time $(T D B)$ and as a function in Barycentric coordinate time $(T C B)$. This is necessary because, till now, in the expression of $T C B-T C G$, the time is $T C B$ but the potential due to all the bodies of the solar system except the Earth and the velocity of the Earth are functions in $T D B$ which involves an error depending on $c^{-4}$ not negligible when we consider the post-Newtonian effects.

\section{References}

Bretagnon, P. (1982) Astron. Astrophys., 114, pp. 278.

Bretagnon, P. and Francou, G. (1988) Astron. Astrophys., 202, pp. 309

Bretagnon, P., Rocher, P. and Simon, J.-L. (1997a) Astron. Astrophys., 319, pp. 305

Bretagnon, P., Francou, G., Rocher, P. and Simon, J.-L. (1997b) Astron. Astrophys., in press.

Fairhead, L. and Bretagnon, P. (1990) Astron. Astrophys., 229, pp. 240.

Standish, E.M. (1982) Astron. Astrophys., 114, pp. 297 